\title{
Casimir Effect in Active Matter Systems
}

\author{
D. Ray $^{1,2}$, C. Reichhardt ${ }^{1}$, and C. J. Olson Reichhardt ${ }^{1}$ \\ ${ }^{1}$ Theoretical Division, Los Alamos National Laboratory, Los Alamos, New Mexico 87545 USA \\ ${ }^{2}$ Department of Physics, University of Notre Dame, Notre Dame, Indiana 46556, USA
}

(Dated: August 20, 2018)

\begin{abstract}
We numerically examine run-and-tumble active matter particles in Casimir geometries composed of two finite parallel walls. We find that there is an attractive force between the two walls of a magnitude that increases with increasing run length. The attraction exhibits an unusual exponential dependence on the wall separation, and it arises due to a depletion of swimmers in the region between the walls by a combination of the motion of the particles along the walls and a geometric shadowing effect. This attraction is robust as long as the wall separation is comparable to or smaller than the swimmer run length, and is only slightly reduced by the inclusion of steric interactions between swimmers. We also examine other geometries and find regimes in which there is a crossover from attraction to repulsion between the walls as a function of wall separation and run length.
\end{abstract}

PACS numbers: $64.75 . \mathrm{Xc}, 47.63 . \mathrm{Gd}, 87.18 . \mathrm{Hf}$

The Casimir geometry consists of two finite parallel plates placed at a fixed distance from each other that experience an attraction due to the confinement of fluctuations in the media between the plates [1]. In the original Casimir effect calculation, electromagnetic vacuum fluctuations produce an attractive force between two metal places in a vacuum. Much later, Casimir forces were experimentally measured 2], and they have recently been studied in a variety of systems in order to understand how to control their magnitude [3] or polarity [4]. Confined classical fluctuations can produce the so-called critical Casimir effect [5 - 7], as first proposed by Fisher and de Gennes near critical demixing in bulk mixtures. The critical Casimir effect has been directly measured [8] and studied in various colloidal systems where it can produce colloidal aggregation [8, 9]. Casimir type effects have also been studied in granular media, where attractive forces arise between objects or plates placed in vibrated or flowing sand 10]. Critical Casimir effects have also been proposed to occur near percolation thresholds 11] and in biological systems such as near fluctuating cellular membranes [12]. The ability to enhance or control such forces can lead to a wide variety of applications in self-assembly, particle transport, and the creation of novel devices.

Strong fluctuations appear in active matter or selfdriven particle systems [13] such as swimming bacteria undergoing run-and-tumble dynamics [14, 15]. Recently a number of non-biological active matter systems have been realized experimentally, including artificial swimmers [16], self-driven colloids [17 19], or light-activated colloidal particles performing a directed random walk [18 20]. Studies of interacting particles undergoing runand-tumble or active Brownian motion show a phase separation phenomenon at large run length or high density in which the particles form dense regions separated by a dilute active gas [20 23]. Monodisperse active particles, such as self-propelled disks, can form intermittent dense patches with crystalline order, termed "living crystals"
[19]. It has been shown that run-and-tumble dynamics and active Brownian motion can be mapped on to each other so that results obtained with one class of system should be generalizible to the other 24$]$.

Here we address the question of whether a Casimirlike attractive force arises between two plates placed in a bath of active particles, and whether such a force can be controlled by changing the plate geometry. There is already some evidence that active matter systems, particularly run-and-tumble particles, can induce forces on objects. One example is the ratchet effect observed for swimming bacteria and run-and-tumble particles in the presence of an array of asymmetric funnels [15, 25 28]. Here, when the active particles run along the walls of the funnel they can escape through easy direction of the funnel or become trapped in a funnel tip. While in contact with a wall, their continued swimming produces forces on the funnel walls. Such induced forces were more clearly demonstrated in systems where an untethered asymmetric sawtooth gear rotates in a preferred direction when placed in a bacterial bath [29, 30], but only if the bacteria are actively swimming. In simulations, asymmetric objects placed in an active matter bath are pushed to produce an active matter-driven micro-shuttle [31, 32].

In the Casimir geometry, there are no sharp corners in which the particles can accumulate; however, we show that confinement effects alone are sufficient to create fluctuation-induced forces. The magnitude of the attractive force increases with increasing run length and can vary over several orders of magnitude. For fixed run length, the force $F$ between the plates as a function of plate spacing $d$ obeys $F(d) \propto \exp (-\lambda d)$, rather than a power law which is usually observed in Casimir geometries [1, 2, 4, [6]. The attractive force arises due to the depletion of the particle density between the plates by a combination of the motion of the particles along the walls and geometric shadowing, both of which increase with increasing run length. We also examine other geometries 


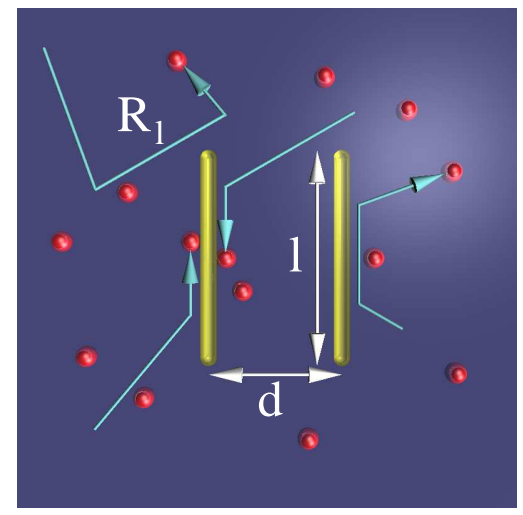

FIG. 1: A schematic of the system containing run-andtumble particles (balls) with some particle trajectories indicated by lines and arrows. The run length is $R_{l}$. The two parallel walls (bars) are of length $l$ and are separated by a distance $d$. When a particle moves along a wall it imparts a force against the wall. We measure the net force $\delta F=\left|F_{\text {out }}\right|-\left|F_{\text {in }}\right|$ for varied $R_{l}$ and $d$, and consider both noninteracting and dilute interacting particles.

and show that it is possible to control the magnitude of the forces and induce a crossover from an attractive to a repulsive force. For the case of infinite walls, we analytically derive the force as a function of the distance between the walls.

Simulation- In Fig. 1 we show a schematic of our system which resides in a two-dimensional (2D) simulation box of size $L_{x} \times L_{y}$ with periodic boundary conditions in the $x$ - and $y$-directions. Unless otherwise noted, we take $L_{x}=120$ and $L_{y}=60$. Within the box are $N$ active particles and two parallel walls of length $l$ separated by a distance $d$. The run-and-tumble particles move in a fixed randomly chosen direction during a running time $\tau$ before undergoing a tumbling event and running again in a new randomly chosen direction. Since we consider the dilute limit where particle-particle interactions are rare and clustering does not occur, for efficiency we use event driven (ED) dynamics simulations. The particles run with speed $v=1$, and we neglect particle-particle interactions. Instantaneous tumble events occur every $\tau$ time units, so during a free run the particle moves a run length of $R_{l}=\tau v$. When a particle encounters a wall, the wall absorbs the component of motion perpendicular to the wall: that is, a particle contacting a wall at an angle $\theta$ moves with velocity $\sin \theta$ along the wall and exerts a force $\cos \theta$ on the wall. If the particle reaches the end of the wall before tumbling, it returns to its original swimming direction. The assumption that run-andtumble particles move along or accumulate at walls has been experimentally confirmed in ratchet geometry [15] and asymmetric gear experiments [29, 30] and observed in simulations [25 27]. We measure the time-averaged force $F_{\text {out }}$ imparted on the outside edges of the walls and $F_{\text {in }}$ imparted on the inside edges. The net force acting to
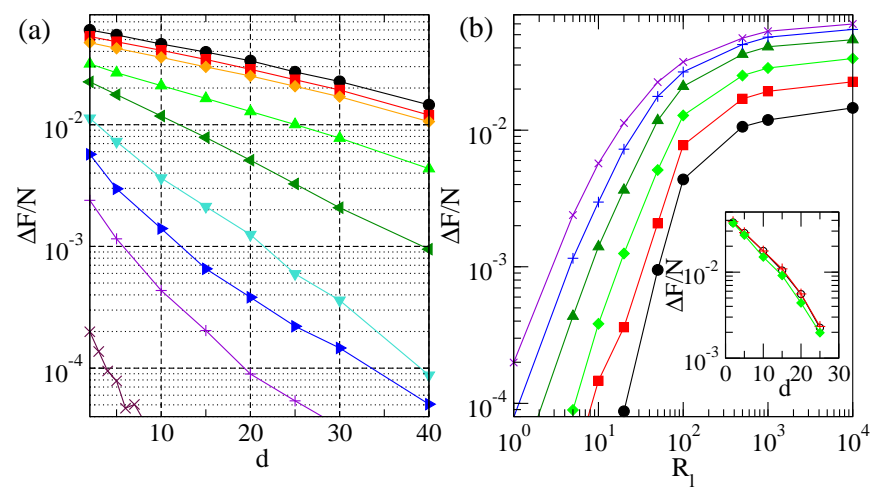

FIG. 2: (a) $\Delta F / N$, the attractive force between the two walls, vs $d$, the distance between the walls, for ED simulations with $l=20$. From top to bottom, $R_{l}=10000,100,500,100$, $50,20,10,5$, and 1 . The curves can be fit by $\Delta F / N \propto$ $A \exp (-\lambda d)$. (b) $\Delta F / N$ vs $R_{l}$ for the same system at $d=2$, $5,10,20,30$, and 40 from top to bottom. For large $R_{l}, \Delta F / N$ saturates. Inset: $\Delta F / N$ vs $d$ for $l=20, R_{l}=20$, and $N=400$ in ED simulations $(\bullet)$ and MD simulations without $(+)$ and with $(\checkmark)$ steric particle-particle interactions. There is only a small shift in $\Delta F / N$ at large $d$ for the interacting system.

bring the walls together or apart is $\Delta F=\left|F_{\text {out }}\right|-\left|F_{\text {in }}\right|$; we report the net force per particle, $\Delta F / N$.

We also confirmed our results using molecular dynamics (MD) simulations with and without particle-particle interactions and find that all of our results are robust for the particle densities we consider. In the MD simulations the dynamics of particle $i$ are obtained by integrating the overdamped equation of motion $\eta d \mathbf{R}_{i} / d t=\mathbf{F}_{i}^{m}+\mathbf{F}_{i}^{s}+\mathbf{F}_{i}^{b}$. Here $\eta=1.0$ is the damping constant and $\mathbf{F}_{i}^{m}$ is the motor force which has a fixed magnitude $F^{m}=1$. During each run time $\tau$ the particle moves a distance $R_{l}=F^{m} \tau$ in the absence of interactions. The particleparticle steric interactions are a stiff-spring repulsion given by $\mathbf{F}_{i}^{s}=\sum_{j \neq i}^{N} k_{s}\left(2 r_{d}-\left|\mathbf{r}_{i j}\right|\right) \Theta\left(2 r_{d}-\left|\mathbf{r}_{i j}\right|\right) \hat{\mathbf{r}}_{i j}$, where $\mathbf{r}_{i j}=\mathbf{R}_{i}-\mathbf{R}_{j}, \hat{\mathbf{r}}_{i j}=\mathbf{r}_{i j} /\left|\mathbf{r}_{i j}\right|, \mathbf{R}_{i(j)}$ is the location of particle $i(j), k_{s}=300$ is the spring constant, and $r_{d}=0.5$ is the particle radius. The particle-barrier interactions $\mathbf{F}_{i}^{b}$ are of a similar form, and are the same as those used in previous simulations 25, 33]. When a particle contacts a wall it moves along the wall with the component of its motor force parallel to the wall and imparts the perpendicular force component to the wall.

Results- In Fig. 2(a) we plot the normalized force $\Delta F / N$ versus $d$ for ED simulations of a system with $l=20$ at run lengths $R_{l}=10000,1000,500,100,50$, 20,5 , and 1 . The magnitude of $\Delta F / N$ increases with increasing $R_{l}$ and we find $\Delta F(d) \propto A \exp (-\lambda d)$, with $\lambda$ decreasing for increasing $R_{l}$. At small runs lengths such as $R_{l}=1, \Delta F / N$ becomes very small, and in the limit of infinitesimal $R_{l}$ or Brownian particles there is no attractive force between the walls. In Fig. 2(b) we plot $\Delta F$ versus $R_{l}$ for the same system at $d=2,5,10,20,30$, 

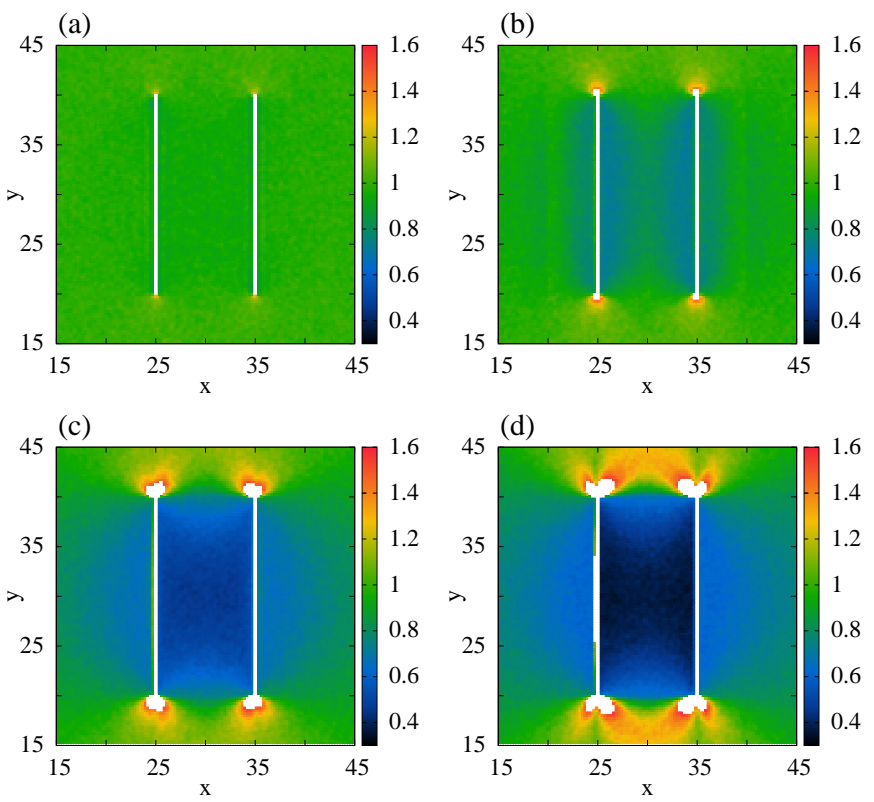

FIG. 3: $\rho(\mathbf{r})$, the spatial distribution of the average particle density, for the system in Fig. 2(a) with $L_{x}=60, l=20$, and $d=10$ at (a) $R_{l}=1$, (b) $R_{l}=5$, (c) $R_{l}=20$, and (d) $R_{l}=50 . \quad \rho$ becomes increasingly depleted between the plates with increasing $R_{l}$, leading to a larger net attractive force between the walls.

and 40 , showing that the attractive force increases with increasing $R_{l}$ and saturates at large $R_{l}$. To verify the robustness of the results, we perform three different types of simulations highlighted in the inset of Fig. 2(b) where we plot $\Delta F / N$ vs $d$ for systems with $N=400, l=20$, and $R_{l}=20$. The results of the ED and MD simulations with noninteracting particles are almost identical. When steric particle-particle interactions are included in the MD simulations, $\Delta F / N$ is nearly unchanged for small $d$ and slightly reduced at larger $d$, and still depends exponentially on $d$. This indicates that our results are robust in the dilute limit. At higher particle densities, phase separation or clustering becomes important if steric particleparticle interactions are included 21-23], a case that we do not consider in this work.

In order to better understand the origin of the attractive force, in Fig. 3 we plot $\rho(\mathbf{r})$, the spatial distribution of the average particle density, from ED simulations with $L_{x}=60, l=20$, and $d=10$ for different values of $R_{l}$. We use the normalization that $\rho=1.0$ in a homogeneous system without walls. For short run lengths $R_{l}=1$ in Fig. 3(a), $\rho \approx 1$ throughout the sample and there is little to no attractive force between the walls. Although $\rho>1$ immediately adjacent to the walls, the density of particles on the inside and outside edges of the walls is virtually identical. At $R_{l}=5$, shown in Fig. 3(b), two trends start to appear. There is a depletion region of lower $\rho$ near the walls which is most pronounced at their centers. In addi-
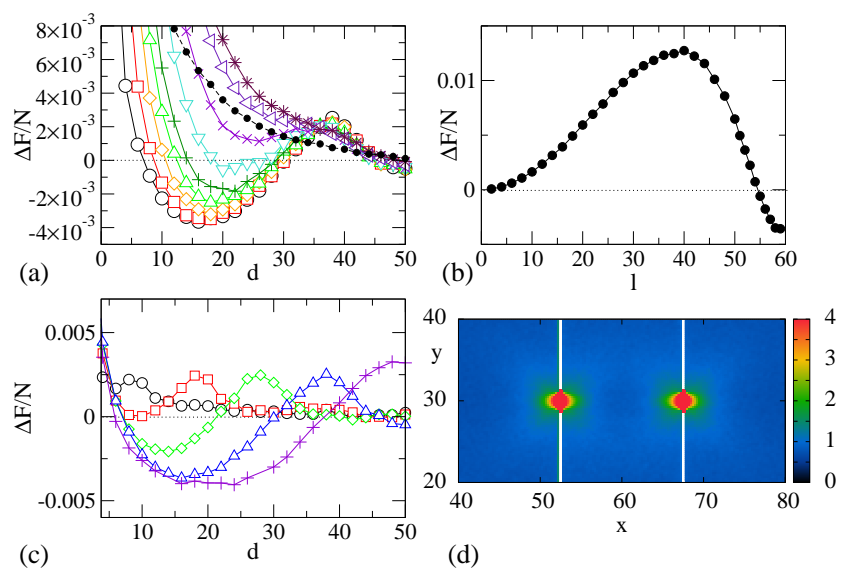

FIG. 4: (a) $\Delta F / N$ vs $d$ for a system with $R_{l}=40$ at $l=20$ $(\bullet), 40(*), 45(\triangleleft), 50(\mathrm{x}), 53(\nabla), 55(+), 56(\triangle), 57(\diamond)$, $58(\square)$ and $59(\bigcirc)$. For $l \geq 55$ there is a regime where the force becomes negative. (b) $\Delta F / N$ vs $l$ for fixed $d=15$ and $R_{l}=40$. Initially, $\Delta F / N$ is attractive and increases before reaching a maximum near $l=40$ and then becoming negative at larger $l$. (c) $\Delta F / N$ vs $d$ for a system with $l=59$ at $R_{l}=10$ $(\bigcirc), 20(\square), 30(\diamond), 40(\triangle)$ and $50(+)$. (d) $\rho(\mathbf{r})$ for a system with $l=58$ at $d=15$.

tion, the higher $\rho$ along the walls produces high density spots at the wall ends where the particles escape from the walls and disperse back into the bulk. The depletion of $\rho$ between the walls is similar to a ray optics shadow effect caused by the finite run length $R_{l}$. Particles exerting forces on the outer edges of the walls can originate from a broad region. In contrast, particles exerting forces on the inner walls originate from a much smaller region. The resulting shadowing effect depletes $\rho$ between the walls. As $d$ decreases, the difference in size between the inner and outer regions grows, leading to an increased shadow effect and hence a stronger $\Delta F$ for smaller $d$. At $R_{l}=20$ in Fig. 3(c), the shadowing effect becomes more pronounced and $\rho$ is strongly reduced between the walls, while in Fig. 3(d), for $R_{l}=50 \rho$ between the walls is even further reduced and the density distribution at the ends of the walls develops additional features due to interference effects. These results show that Casimir attractive forces can arise in active matter systems and that they can be controlled by modifying the run length or persistence length of the swimming particles.

Reverse Casimir Effect- We next consider the effect of changing $l$, the wall length. Since we have periodic boundary conditions, at large $l$ the system is better described as consisting of apertures of width $w=L_{y}-l$, as shown in Fig. 4(d) for a system with $l=58$. In Fig. 4(a) we plot $\Delta F / N$ versus $d$ for a system with $R_{l}=40$ at $l=20,40,45,50,53,55,56,57,58$, and 59. For $l<55$, the force is attractive for all $d$. For $l \geq 55$, there is a range of $d$ for which $\Delta F / N$ becomes negative, while at larger $d$ the force becomes positive again. The magni- 
tude of the negative force depends on $l$, with the largest negative forces appearing for the smallest values of $w$. In Fig. 4(b) we plot $\Delta F / N$ versus $l$ for a system with $d=15$ and $R_{l}=40$. Here $\Delta F / N$ is nearly zero at small $l$ and increases with increasing $l$ up to $l \approx 40$ due to the shadowing effect. For $l>40, \Delta F / N$ decreases rapidly and becomes negative, competing with the shadowing effects that tend to increase the attractive force. The drop arises from a particle trapping effect that occurs as the system enters the aperture limit. The particles moving along an outside wall return to the bulk upon reaching the wall end when $l$ is small, but for large $l$ they instead are trapped by the interior region, raising the interior density and producing a net repulsive force between the two walls. The trapping effect becomes more prominent with decreasing $w$. In Fig. 4 (a), where $R_{l}=40$, for $l \geq 53$ and $d<30$ the particles in the interior region spend most of their time running along the interior walls, producing a repulsive force. For $d>30$ the particles have a chance to turn away from the inside wall during their run and avoid striking it, so the force becomes attractive again.

In Fig. 4(c) we plot $\Delta F / N$ versus $d$ for systems with different $R_{l}$. For the short run length of $R_{l}=10, \Delta F / N$ is always positive, but as $R_{l}$ increases, the force drops below zero over a region that increases with increasing $R_{l}$, and returns to positive values at higher values of $d$. The resulting shape of $\Delta F / N(d)$ resembles an atomic interaction force curve, with a stable characteristic distance determined by the point at which $\Delta F / N$ crosses zero with positive slope, suggesting that freely moving walls could be stabilized at this spacing. The spacing can be adjusted by varying $R_{l}$ in order to move the zero point of $\Delta F / N$ to the desired distance, opening the possibility that active matter could be used to self-assemble passive objects not merely to the aggregation point of zero spacing, but also to a finite spacing set by $R_{l}$. These results show that by varying the geometry it is possible to achieve detailed control over the magnitude of the fluctuation-induced forces.

Infinite Walls- We have also considered the case of infinite walls that confine the particles to an interior region, producing only an outward force. Such a system could be created by trapping bacteria or active matter particles in a confined geometry and measuring the force exerted on the walls. We consider both one-dimensional (1D) and $2 \mathrm{D}$ infinite wall systems and measure the force for different wall spacings $d$, with the interior particle density held fixed. In the limit of Brownian particles, $\Delta F$ would be independent of $d$. In Fig. 5(a) we plot the outward force $-\Delta F / N$ versus $d / R_{l}$ for a $1 \mathrm{D}$ system. When $l$ is infinite, the only length scales are $d$ and $R_{l}$. The fraction of time a particle spends traveling along the walls is unchanged if both these lengths are scaled by the same factor, and so the force must be a function of the ratio $d / R_{l}$. Fig. 5 shows that the curves for various $R_{l}$ can be collapsed to the form $F(x)=1 /(1+x)$ with $x \equiv d / R_{l}$, which can be
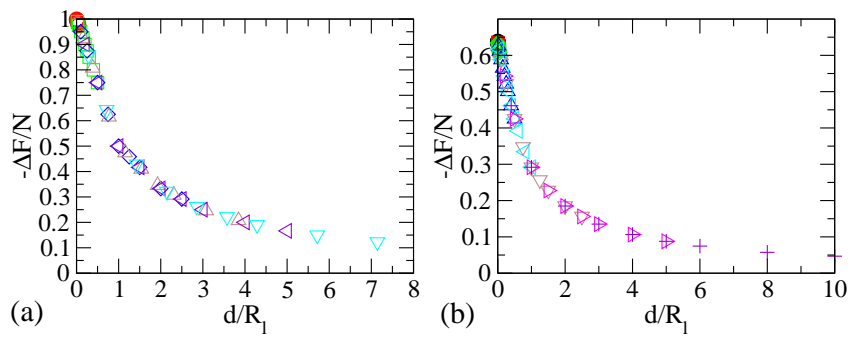

FIG. 5: The outward force $-\Delta F / N$ vs $d / R_{l}$ for a system with infinite walls where the active particles are confined to move only between the walls from ED simulations. The particle density between the walls is held constant. (a) 1D systems with $R_{l}=1000(\bigcirc), 100(\square), 20(\diamond), 13(\triangle), 10(\triangleleft)$, and $7(\nabla)$. The curves can be collapsed to the functional form $\Delta F(d)=R_{l}\left(R_{l}+d\right)^{-1}$. (b) $2 \mathrm{D}$ systems with $R_{l}=10000(\bigcirc)$, $1000(\square), 500(\diamond), 100(\triangle), 50(\triangleleft), 20(\nabla), 10(\triangleright)$, and $5(+)$. A similar data collapse occurs.

derived from elementary considerations. For simplicity, consider integer $x$ and measure length in units of $R_{l}$ and time in units of $\tau$. The particle moves in 1D along $y$ with walls located at $y=0$ and $y=x$. During each run time, the particle moves a distance \pm 1 along $y$. To calculate the relative amount of time a particle spends at a wall, we note that a particle emerges into the bulk by tumbling away from a wall; taking this to be the $y=0$ wall, the particle takes one step to $y=1$. We then need to calculate the expected time $t_{b}$ required for the particle to either return to $y=0$ or reach $y=x$. This is the "gambler's ruin" problem, with the well-known solution of $(x-1)$. Counting the first step into the bulk, the expected number of steps in the bulk between wall motions is just $x$, so $t_{b}=x$. To determine $t_{w}$, the amount of time the particle spends at the wall after reaching it, we note that each tumble provides a 50:50 chance of escaping the wall, giving a probability $p=\frac{1}{2}$ that $t_{w}=0, p=\frac{1}{4}$ that $t_{w}=1$, and so on. The expected value of $t_{w}$ is easily found to be $t_{w}=1$. Therefore, the ratio of $t_{w}$ to the total time is $t_{w} /\left(t_{w}+t_{b}\right)=1 /(1+x)$. This equals the time-averaged force on the walls since in the 1D scenario, the force on a wall is 1 when a particle is present at the wall and 0 otherwise. For noninteger $x$, it is not too difficult to derive a more general formula $\frac{2-\frac{x}{\operatorname{ceil}(x)}}{1+\operatorname{ceil}(x)}$ where $\operatorname{ceil}(x)$ is the smallest integer $\geq x$.

In Fig. $5(\mathrm{~b})$ we plot $-\Delta F / N$ for a $2 \mathrm{D}$ system. Here we could not derive a fully analytic solution; however, we obtain a fit of $F(x)=a /(1+b x)$ with $a=2 / \pi$ and $b \approx 1.25$. The value for $a$ is exact and arises because in $2 \mathrm{D}$ all angles of incidence are possible rather than just normal incidence. Since the angle distribution is uniform, the resulting force on the wall is lessened by a factor of $\frac{1}{\frac{\pi}{2}-0} \int_{0}^{\pi / 2} \cos \theta \mathrm{d} \theta=\frac{2}{\pi}$. We cannot calculate $b$ exactly, but it can be understood heuristically: compared to the above 1D derivation, the particle spends 
more time in the bulk because only the $y$ component of its motion moves it towards a wall. Therefore the effective step size is reduced from 1 and $t_{b}$ is increased, giving $b>1$. When steric particle-particle interactions are included, the forces on the walls are reduced; however, in the dilute limit these results should hold and should be readily testable in experiments.

Summary - We have shown that in a Casimir geometry of two parallel walls placed in a bath of run-and-tumble active particles, a robust attractive force arises between the walls due to a combination of the particles moving along the walls and a geometric shadowing effect that depletes the particle density between the walls. The depletion becomes more pronounced for increasing particle run length or reduced wall spacing, increasing the magnitude of the force. Our results are robust against the inclusion of steric particle-particle interactions in the dilute limit. We also find that for other geometries such as two walls containing small apertures, a particle trapping effect can produce repulsive forces between the walls. Our results show that active matter systems can exhibit a rich variety of fluctuation-induced forces between objects, and these effects may be useful for applications such as self assembly or particle transport.

We thank D. Dalvit and L. Lopatina for useful discussions. This work was carried out under the auspices of the NNSA of the U.S. DoE at LANL under Contract No. DE-AC52-06NA25396.

[1] H.B.G. Casimir, Proc. K. Ned. Akad. Wet. 51, 793 (1948).

[2] S.K. Lamoreaux, Phys. Rev. Lett. 78, 5 (1997).

[3] F. Intravaia, S. Koev, I.W. Jung, A.A. Talin, P.S. Davids, R.S. Decca, V.A. Aksyuk, D.A.R. Dalvit, and D. Lopez, Nature Commun. 4, 3515 (2013).

[4] J.N. Munday, F. Capasso, and V.A. Parsegian, Nature (London) 457, 170 (2009).

[5] M.E. Fisher and P.G. de Gennes, C. R. Acad. Sci. Ser. B 287, 207 (1978).

[6] C. Hertlein, L. Helden, A. Gambassi, S. Dietrich, and C. Bechinger, Nature (London) 451, 172 (2008).

[7] A. Hanke, F. Schlesener, E. Eisenriegler, and S. Dietrich, Phys. Rev. Lett. 81, 1885 (1998); A. Gambassi, J. Phys.: Conf. Ser. 161, 012037 (2009).

[8] D. Bonn, J. Otwinowski, S. Sacanna, H. Guo, G. Wegdam, and P. Schall, Phys. Rev. Lett. 103, 156101 (2009); A. Gambassi and S. Dietrich, Phys. Rev. Lett. 105, 059601 (2010).

[9] S.J. Veen, O. Antoniuk, B. Weber, M.A.C. Potenza, S. Mazzoni, P. Schall, and G.H. Wegdam, Phys. Rev. Lett. 109, 248302 (2012); V.D. Nguyen, S. Faber, Z. Hu, G.H. Wegdam, and P. Schall, Nature Commun. 4, 1584 (2013).

[10] C. Cattuto, R. Brito, U.M.B. Marconi, F. Nori, and R. Soto, Phys. Rev. Lett. 96, 178001 (2006); Y.Y. Villanueva, D.V. Denisov, S. de Man, and R.J. Wijngaarden, Phys. Rev. E 82, 041303 (2010).
[11] N. Gnan, E. Zaccarelli, and F. Sciortino, Nature Commun. 5, 4267 (2014).

[12] B.B. Machta, S.L. Veatch, and J.P. Sethna, Phys. Rev. Lett. 109, 138101 (2012).

[13] S. Ramaswamy, Annu. Rev. Condens. Matter Phys. 1, 323 (2010); M.C. Marchetti, J.F. Joanny, S. Ramaswamy, T.B. Liverpool, J. Prost, M. Rao, and R.A. Simha, Rev. Mod. Phys. 85, 1143 (2013).

[14] H.C. Berg, Random Walks in Biology (Princeton University Press, Princeton, 1983).

[15] P. Galajda, J. Keymer, P. Chaikin, and R. Austin, J. Bacteriol. 189, 8704 (2007).

[16] R. Dreyfus, J. Baudry, M.L. Roper, M. Fermigier, H.A. Stone, and J. Bibette, Nature (London) 437, 862 (2005).

[17] W.F. Paxton, K.C. Kistler, C.C. Olmeda, A. Sen, S.K. St. Angelo, Y.Y. Cao, T.E. Mallouk, P.E. Lammert, and V.H. Crespi, J. Am. Chem. Soc. 126, 13424 (2004); J.R. Howse, R.A.L. Jones, A.J. Ryan, T. Gough, R. Vafabakhsh, and R. Golestanian, Phys. Rev. Lett. 99, 048102 (2007); R. Golestanian, Phys. Rev. Lett. 102, 188305 (2009).

[18] G. Volpe, I. Buttinoni, D. Vogt, H.-J. Kummerer, and C. Bechinger, Soft Matter 7, 8810 (2011).

[19] J. Palacci, S. Sacanna, A.P. Steinberg, D.J. Pine, and P.M. Chaikin, Science 339, 936 (2013).

[20] I. Buttinoni, J. Bialke, F. Kümmel, H. Löwen, C. Bechinger, and T. Speck, Phys. Rev. Lett. 110, 238301 (2013).

[21] J. Tailleur and M.E. Cates, Phys. Rev. Lett. 100, 218103 (2008); A.G. Thompson, J. Tailleur, M.E. Cates, and R.A. Blythe, J. Stat. Mech.: Theory Exp. 2011, P02029 (2011).

[22] J. Bialke, T. Speck, and H. Löwen, Phys. Rev. Lett. 108, 168301 (2012).

[23] Y. Fily and M.C. Marchetti, Phys. Rev. Lett. 108, 235702 (2012); G.S. Redner, M.F. Hagan, and A. Baskaran, Phys. Rev. Lett. 110, 055701 (2013); C. Reichhardt and C.J. Olson Reichhardt, arXiv:1402.3260

[24] M.E. Cates and J. Tailleur, EPL 101, 20010 (2013).

[25] M.B. Wan, C.J. Olson Reichhardt, Z. Nussinov, and C. Reichhardt, Phys. Rev. Lett. 101, 018102 (2008); C. Reichhardt and C.J. Olson Reichhardt, Phys. Rev. E 88, 062310 (2013).

[26] J. Tailleur and M. E. Cates, Europhys. Lett. 86, 60002 (2009).

[27] L. Angelani, A. Costanzo, and R. Di Leonardo, EPL 96, 68002 (2011); V. Kantsler, J. Dunkel, M. Polin, and R.E. Goldstein, Proc. Natl. Acad. Sci. (USA) 110, 1187 (2013); I. Berdakin, Y. Jeyaram, V.V. Moshchalkov, L. Venken, S. Dierckx, S.J. Vanderleyden, A.V. Silhanek, C.A. Condat, and V.I. Marconi, Phys. Rev. E 87, 052702 (2013).

[28] M.E. Cates, Rep. Prog. Phys. 75, 042601 (2012).

[29] L. Angelani, R. Di Leonardo, and G. Ruocco, Phys. Rev. Lett. 102, 048104 (2009); R. Di Leonardo, L. Angelani, D. Dell'Arciprete, G. Ruocco, V. Iebba, S. Schippa, M.P. Conte, F. Mecarini, F. De Angelis, and E. Di Fabrizio, Proc. Natl. Acad. Sci. U.S.A. 107, 9541 (2010).

[30] A. Sokolov, M.M. Apodaca, B.A. Grzybowski, and I.S. Aranson, Proc. Natl. Acad. Sci. U.S.A. 107, 969 (2010).

[31] N. Koumakis, A. Lepore, C. Maggi, and R. Di Leonardo, Nature Commun. 4, 2588 (2013).

[32] L. Angelani and R. Di Leonardo, New J. Phys. 12, 113017 (2010). 
[33] J.A. Drocco, C.J. Olson Reichhardt, and C. Reichhardt, Phys. Rev. E 85, 056102 (2012). 